\title{
Multihoming in Nested Mobile Networking
}

\author{
Nicolas Montavont \\ Louis Pasteur University \\ LSIIT - CNRS 7005 \\ Bld Sebastien Brant \\ 67400 Illkirch-Graffenstaden - France \\ montavont@dpt-info.u-strasbg.fr
}

\author{
Thierry Ernst \\ Jun Murai Lab. Keio University \\ K2 Town Campus \\ 1488- Ogura, Saiwai-ku, Kawasaki \\ Kanagawa 212-0054 - Japan \\ ernst@sfc.wide.ad.jp
}

\author{
Thomas Noel \\ Louis Pasteur University \\ LSIIT - CNRS 7005 \\ Bld Sebastien Brant \\ 67400 Illkirch-Graffenstaden - France \\ noel@dpt-info.u-strasbg.fr
}

\begin{abstract}
The management of network mobility raises new issues in host mobility: how to maintain IPv6 communication to all nodes inside a mobile network, how to allow nodes to choose the best default router in a multihomed mobile network. In the context of the Nautilus working group within the WIDE community, this article presents what is a mobile network and what is the implications of multihoming and the aggregation of several mobile networks. The paper also presents an optimization in Router Advertisement to allow hosts to discover the hierarchy of Mobile Routers in nested mobile networks.
\end{abstract}

\section{Introduction}

Nowadays wireless technologies are widely used in IPv6 communication. WLANs technologies including IEEE 802.11 series and Bluetooth, 3GPP technologies such as GPRS and 3GPP2 such as CDMA2000 and UMTS allow mobile equipments to be connected everywhere and at anytime. When considering the mobility management among access networks, two types of mobility can be observed: on one hand, single mobile hosts moving between IPv6 subnets need to maintain their sessions with the global Internet, even after tough they changed their IPv6 address. On the other hand, an entire mobile network (MNet) may change its point of attachment to the Internet. In this case, all communication between the nodes inside the MNet and the global Internet must be maintained.

The management of host and network mobility is currently under discussion at the IETF $^{1}$. Host mobility is managed by the Mobile IPv6 [7] protocol and is standardized in the IETF's MIP6 working group. Network mobility has recently been introduced at the IETF in 2001 and gave birth to new working group called NEMO (NEtwork MObility) set up in fall 2002. For the moment, the NEMO working group works on a basic specification to support network mobility: NEMO Basic Support [4]: the movement of the MNet is transparent to the nodes inside the MNet and their packets are tunneled in both directions between the mobile router (MR) and its home agent (HA). Route optimization for nodes inside the MNet is not yet considered by the working group although several proposals have already been submitted.

Furthermore, when a host has several IPv6 addresses to choose between, it is said multihomed [9]. Multihoming offers three main benefits to hosts: it allows route recovery on failure, redundancy and load-sharing. However, for the moment there are no requirements nor protocol defining how to use several interfaces inside a MNet.

The Nautilus project [2] recently set up within the WIDE community in Japan [1] is working on this issue. Nautilus's overall goal is to demonstrate the reality of IPv6 mobility. For this to happen, several technologies must be integrated: host mobility, network mobility, multihoming, seamless mobility, security, access control and applications. Host mobility and network mobility are the core activities.

\footnotetext{
${ }^{1}$ Internet Engineering Task Force
} 
Multihoming is necessary to provide constant access to the Internet and to enhance the overall connectivity of hosts and MNet, whereas seamless mobility is necessary to enhance the performance of handoffs. Security and access control are required to allow deployment at a commercial level. Regarding this lattest activity, Nautilus is just seeking to integrate the minimum mechanisms required to convince commercial players that IPv6 mobility is a reality, while applications are necessary to demonstrate the usefulness and readiness of IPv6 mobility. Each activity follows a roadmap that describes the steps to select, implement and validate the required protocols in the activity. Implementations are being validated on an indoor testbed, while mature features are demonstrated in an operational testbed.

Right now, only host mobility (i.e. Mobile IPv6) and companion IPv6 protocols developped by other WIDE projects (mainly Kame and Usagi) have reached this level of maturity. One of the current Nautilus activity is thus the developpement of an operational testbed to demonstrate the usefulness and readiness of the Mobile IPv6 protocol. Mobile IPv6 and applications to benefit the mobility feature are being developped for a PDA running Linux. IPv6 applications include web browsing, emailing, and voice over IP. This PDA will be distributed to WIDE members and statistics will be collected to assest the performance and use of Mobile IPv6. Network mobility and multihoming features will be added later once the needed protocols are implemented and validated under the indoor testbed. Then, and in order to demonstrate its operational value, dynamic security and access control mechanisms will be brought to the users. Performance mechanism such as seamless mobility and route optimization will also be considered.

Other current activities of the Nautilus project cover the implementation of NEMO Basic Support and active discussions at the IETF turning around multihoming in both MIP6 and NEMO working groups $[9,3,10]$

In the scope of this article, we investigate multihoming in mobile network, especially in nested MNet (aggregated mobile networks). In the next section we introduce the terminology related to network mobility and multihoming. Next we list different issues for nodes inside multihomed netsted MNets. Then we present a new option in Router Advertisements to help nodes to discover the MR hierarchy. We then conclude this paper with future work going to be done in this topic.

\section{Terminology}

Terms referencing mobile networking are defined in $[8$, 6]. A Mobile Network (MNet) is a set of nodes which can simultaneously change their point of attachment to the Internet. A MNet is composed by one or more IP-subnet(s) and is viewed as a single unit. It is connected to the In- ternet by means of Mobile Routers (MRs). MRs have one or more egress interfaces connected to the global Internet. Nodes inside the MNet are called Mobile Network Nodes. Their point of attachments inside the MNet is not mandatory fixed.

A Mobile Network is said nested when one of the egress interfaces of the MNet is connected to another MNet. When a MNet is getting attached to another MNet, the aggregated hierarchy of MNet becomes a single nested MNet. In the following subsections, we define new terms related to this concept.

\subsection{Definition of terms}

The following defintions are related to network mobility in general:

- Mobile Router (MR): A router capable of changing its point of attachment to the network, moving from one link to another link. It acts as a gateway between an entire MNet and the rest of the Internet.

- Egress interface: One of the MR's interfaces used to forward packets upstream to the rest of the Internet. This interface is either connected to a home link or visited link.

- Ingress interface: one of the MR's interface used to forward packets downstream to the MNNs. It is connected to a mobile network link (see below).

- Mobile network link (MNet link): A mobile network link is a link internal to the mobile network. At least one prefix, or a part of one prefix announced on this link is advertised by a MR.

- Mobile Network Node (MNN): Any node (either a host or a router) located within a MNet, either permanently or temporarily. Such a MNN can be a mobile node (VMN or LMN), a fixed node (LFN), or even a MR (i.e. a sub-MR, see below).

- multi-homing: the multihoming concept may apply either to a MNN, a MR or the entire MNet. A MR is said multihomed when (considering its egress interface(s)):

- multi-prefixed: multiple prefixes are advertised on the link(s) the egress interface(s) is (are) connected to.

- multi-interfaced: multiple egress interfaces to choose between, on the same link or not.

- multi-linked: multiple links to choose between (just like multi-interfaced but all egress interfaces are NOT connected to the same link) 
- multi-sited: when using IPv6 site-local address and attached to different sites.

The following definitions are specific to netsted mobile networks:

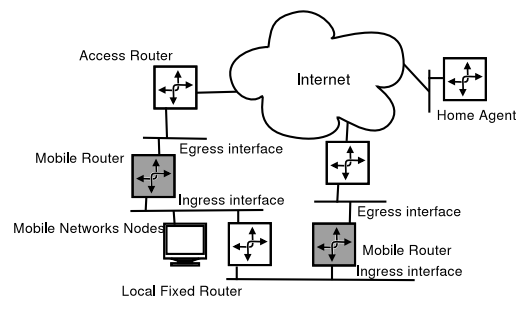

Figure 1. Non-nested Mobile Network

- Non-nested mobile network: indivisable mobile network. Each egress interface of MR(s) composing a non-nested mobile network is directly attached to the Internet, without using an intermediate mobile network. Mobile network represented in Figure 1 is a non-nested MNet.

- Root-related terminology: For a mobile network to be root-mobile network, one of its MR must have a direct access to the Internet. A root-MR is a MR which belongs to a root-mobile network and has anegress interface directly connected to the Internet.

- Parent-related terminology: A parent-mobile network is a MNet used by another MNet to access Internet,i.e. one of the prefix sent by a MR of the parent mobile network is used by another MR to configure a Care-of address on one of its egress interface. A MR inside a parent-MNet is a parent-MR.

- Sub-related terminology: a sub-mobile network is a mobile network which uses another MNet, higher in the hierarchy of the entire nested MNet, to access the Internet. One of the egress interface of a MR belonging a sub-mobile network is connected to another MNet. Such a MR with an egress interface conneted to another MNet is called sub-MR.

- Multi-root nested mobile network: a nested mobile network which has more than one root-non-nested mobile network. A multi-root nested mobile network is at least composed of two root-MRs in two different mobile network entities.

\subsection{Multihoming and nested mobility}

The definition of mutlihomed MNet rises of the above conditions: a MNet (respectively a nested MNet) is said multihomed when it has either a multihomed MR (resp. a multihomed root-MR) or more than one MR (resp. rootMR).

Multihoming in MNet offers useful properties to MNNs. References $[3,10]$ list potential multihomed configurations for MNets and explains the different problems and advantages some configurations may introduce. Multi-homing offers three main abilities to the MNet: it allows route recovery on failure, redundancy and load-sharing between MRs (or between MRs'interfaces). However, for the moment there are no requirements nor protocol defining how to use several egress interfaces inside a MNet.

In nested MNet, the hierarchy of MRs increase the complexity of the route and/or router selection for MNNs. Each level of a MNet implies the usage of a new tunnel between the MR and its home agent. Thus if a MNN connects to a sub-MNet which is also a sub-MNet, packets from the MNN will be encapsulated three times. We will see in the next section how to prevent MNNs to use themost encapsulated route for their packets.

\subsection{Aggregated Mobile Networks}

When an entire MNet changes one of its point of attachment to the Internet, it can attach to a fixed link, or to another MNet. In the second case, the aggregation of the two connected MNet becomes a nested MNet. Let us consider non-nested MNet 1 and non-nested MNet 2 as represented in Figure 2. We then discuss the aggregation of these two MNets.

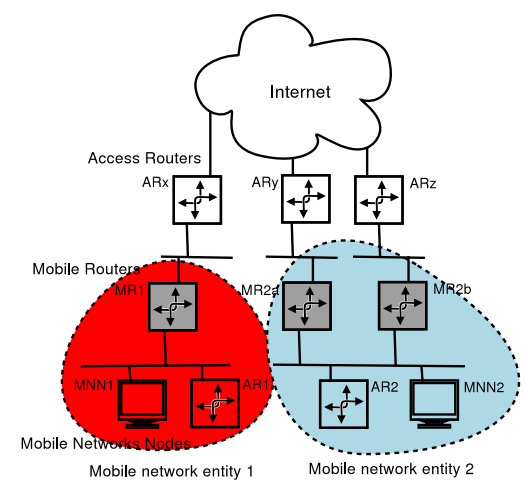

Figure 2. Initial Mobile Networks 1 and 2

\subsubsection{MR2a attaches to AR1}

Let us consider that MR2a changes its point of attachment to the Internet and connects to AR1, as represented in Figure 3, resulting in a nested MNet. The resulting MNet has the following properties: 


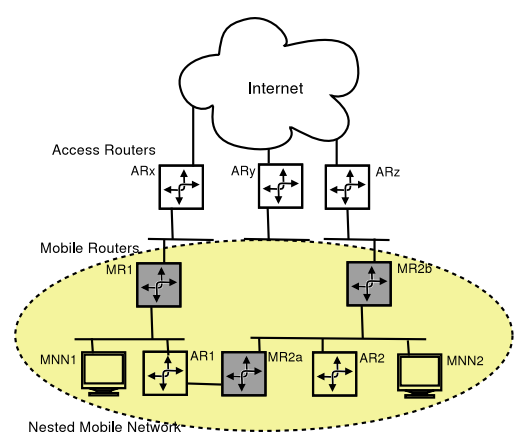

Figure 3. Nested Mobile Network when $\mathbf{M R}_{2} a$ connects to $\mathrm{AR}_{1}$

- The nested MNet is multihomed since it has now two root-MRs (MR1 and MR2b). The non-nested MNet2 is also multihomed as before the aggregation, since both MR2a and MR2b are giving internet access to MNNs of MNet 2. The non-nested MNet 1 remains not multihomed, since only one MR (MR1) is available for MNNs of MNet 1.

- The non-nested MNet 2 is a sub-MNet of non-nested MNet 1 since the Care-of address of MR2a is built from a prefix adverised in MNet 1 . In symetric, the non-nested MNet 1 is the parent-MNet of non-nested MNet 2.

- MR2b and MR1 are root-MRs of the nested MNet and MR2a is a sub-MR of the nested MNet.

\subsubsection{MR1 attaches to AR2}

Let us consider that MR1 changes its point of attachment to the Internet and connects to AR2, as represented in Figure 4. Then the resulting MNet is a nested MNet which has the following properties:

- The nested MNet is multihomed since MR2a and MR2b have an internet access without using another MNet. The non-nested MNet 2 remains multihomed, for the same reason (MR2a and MR2b belong to MNet 2. The non-nested MNet 1 remains not multihomed, since MR1 is the only MR for this entity and is not multihomed itself.

- The non-nested MNet 1 has became the sub-MNet of MNet 2, since it gets its Care-of address from MNet2. Moreover MNet 2 is both the parent-MNet of MNet 1 and the single root-MNet of the nested MNet.

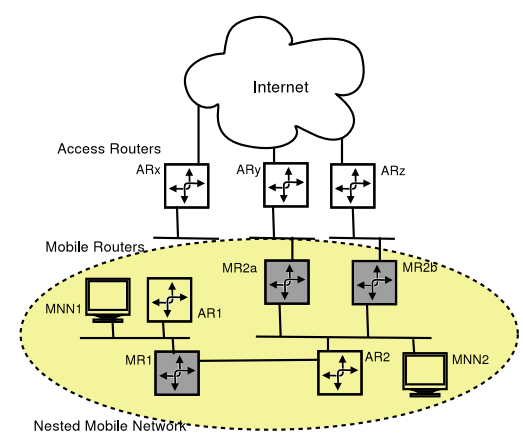

Figure 4. Nested Mobile Network when $\mathrm{MR}_{1}$ connects to AR2

- MR1 is a sub-MR of the nested MNet and MR2a and MR2b are both root-MRs of the nested MNetand parent-MRs of MR1.

\section{MNNs issues in multihomed nested mobile networks}

Currently, route optimization is not defined in NEMO Basic Support [4]. The current point of attachment of the entire MNet is hidden by MR; MRs announce their own prefix obtained on their home network and use a bidirectional tunnel with their home agent for all packets from and to MNNs. Therefore all packets from MNNs will be tunneled to the home agent of the respective MR, and therefrom routed to the relevant $\mathrm{CN}$.

When a MNet is multihomed, the entire MNet benefits of having multiple egress interfaces. However, according to the topology, any MNN inside a mutlihomed MNet is not necessary multihomed; For instance, when MR1 connects to LFR2 (see figure 4), a MNN connected on MR1 link is not multihomed since it has only one prefix on the link.

According to the MNet topology and the point of attachment of MNNs inside the MNet, MNNs may hear different prefixes. When the MNet where the $\mathrm{MN}$ is connected to is multihomed, the MN may have the choice of its default router. Reference [5] introduces new options in Router Advertisement to allow any node on a link to choose between several routers. This option mainly consists of a 2-bits flag that indicates the preference of the router (low, medium or high). Furthermore, the same flag can be set in the Route Information option indicating the preference of a specific prefix. Therefore, any node can determine its best default router(s) according to a given destination and its best router for default, which will be used by default. 
However this preference is only useful in a flat topology; It gives a way to the node to choose between different access routers advertising prefixes on the node link. But if the node is inside a hierarchical topology (some access routers are not at the same level) the node can not be aware about the level of each access router. As we will see in the next subsection, this may lead to an important overhead in nested MNet, where there is a hierarchy of MRs.

\subsection{Overhead introduced aggregated nested mobile network}

In the instance given section 2.3.1 and illustrated in Figure 3, we assume that each MR set up a bidirectional tunnel with their respective home agent. Three different home agents are therefore involved. The movement of MR is fully transparent to MNNs behind MRs. Although MNNs may notice a change in bandwidth, MNNs do not have any indication that MR2a connects to AR1. However, packets sent from MNNs which use MR2a as default router, will be tunneled twice: once between MR2a and its home agent and then another time to the home agent of MR1. Only after the packets will reach the correspondant of the MNNs. This tunneling overhead would furher increase when there are more levels of nesting. If the MNNs on link 2 are able to know the hierarchy of MRs, they may choose to use MR2b instead of MR2a, because MR2b is directly connected to the Internet. The aim of the following sections is to set mechanisms allowing router selection for MNNs.

\section{Route selection in multihomed nested mobile networks}

In order to provide router selection for MNNs inside a multihomed nested MNet, we propose two kind of mechnanisms. The first one is only performed by MRs and is fully transparent to MNNs. The second one is done to allow MNNs to choose their default MR according to the information supplied in Router Advertisements.

\subsection{New hierarchical option in Router Advertise- ment}

We propose to add a new option in Router Advertisement to distribute the knowledge of the MR hierarchy inside a nested MNet. This option allows any receiving MNN to deduce the level of nesting. This option is shown in Figure 5.

- MR: if the MR flag is set, the flag indicates that the sending access router is a MR. Otherwise, the sending node is a fixed access router.

- Level: gives the level in the hierarchy of the sender of this Router Advertisement. 0 is for a root-MNet.The

\begin{tabular}{|l|c|c|c|}
\hline Type & Length & M & Reserved \\
\hline \multicolumn{2}{|c|}{ Level } & \multicolumn{2}{|c|}{ Reserved } \\
\hline
\end{tabular}

\section{Figure 5. New option in Router Advertise- ment: Hierarchical MR}

lower a MR is in the hierarchy, the higher its level number is.

When an access router (of the Internet) receives a Router Advertisement with the MNet hierarchy option set, it adds the same option within its own Router Advertisements. If it is not a MR or is a MR connected to its home network, it must indicate in the option that it is not a MR (MR flagto 0 ). When a MR away from home receives this option, it increments the level and adds the option in its own Router Advertisements. If a MR receives a Router Advertisement without the option, it adds a new option in its own Router Advertisment with 0 as level number (root-MR).

This option is useful both for MRs and for other MNNs. We will see in the next subsections how to use this option.

\subsection{Network-controlled route selection}

Let us assume the nested MNet represented in Figure 3,when MR2a connects to AR1. Both MR2a and MR2b are able to deduce that there is another MR on the samelink. Furthermore, each MR can determine the level of the other MR thanks to the MR option included in Router Advertisement. MR2b is on the level 0 and MR2a is on the level 1 because its egress interface is connected to MNet1. Then, they can configure their preferences as defined in [5]. For example, MR2a can set a low preference while MR2b a medium or a high preference on the prefix. This can help MNNs to choose their best default router andtheir best route for default, without having to understand the new option defining the hierarchy of the nested MNN.

\subsection{MNNs-controlled route selection}

When a MNN is connected to a link whereas several MRs announce a prefix, it uses the preference field of Router Advertisement to choose its default router. Then MNNs may also use the hierarchical option (defined in subsection 4.1) contained in Router Advertisement tolearn the MR hierarchy.

When a MNN receives several Router Advertisements, it may decide which will be its default router. If the Hierarchical MNet option is present, the MNN may use it to make a decision. In the case represented on Figure 3, a MNN attached to the link 2 would choose MR2b rather than MR2a 
because it is able to determine that MR2a is connceted to Internet via a non-nested MNet while MR2b is directly connected to the Internet. Therefore the MNN knows that if MR2b is used for its packets, the packets will only be tunneled to the home agent of MR2b.

Other mechanisms could be used by MNNs to help indefault router selection, such as the delay between each MR and its home agent. But this kind of optimization is currently out of scope of this article and is under work.

\section{Conclusion}

Network mobility is a new wide research area that raise new issues. In addition to the management of the mobility of all nodes inside a mobile network, nested mobile network and multihoming are characteristics to be considered as well for basic support as for optimization. In this paper, we investigated the multihoming issue in nested mobile networks. We first presented the architecture and the terminology used to describe mobile networks. Then we raised issues pertaining to MNNs inside multihomed nested mobile networks. Such issues include the fact that packets can be encapsulated several times before being sent to correspondents. Then we propose a new option in Router Advertisements that allows any receiving node to discover the hierarchy of MRs. On one hand, it allows MRs to know their level in a nested mobile network and to configure their Router Advertisement accordingly. On the other hand, it helps MNNs to choose their default router.

Multihoming will certainly be the most important issue in the mobility management in the coming years. With the recent deployment of different wireless technologies and the new version of the IP protocol, almost all nodes will be multihomed. New protocols and mechanisms are required to benefit of the multihoming feature, such as the simultaneous use of several interfaces or the definition of the best access router identification algorithm in a mobile network. Our future work is to define new ways for MNs and MNNs to benefit of the multihoming feature and to allow an efficient share of the load inside nested mobile networks, based on several parameters such as the wireless technology used, the speed of the movement, the available bandwidth, etc.

\section{References}

[1] WIDE: Widely Integrated Distributed Environment, June 2002. http://www.wide.ad.jp.

[2] Nautilus6 Working Group at WIDE, web page, April 2003. http://www.nautilus6.org.

[3] J. Charbon, C.-W. Ng, K. Mitsuya, and T. Ernst. Evaluating Multi-homing Support in NEMO Basic Solution, work in progress, Internet Engineering Task Force draft-charbonnemo-multihoming-evaluation-00.txt, July 2003.
[4] V. Devarapalli, R. Wakikawa, A. Petrescu, and P. Thubert. NEMO Basic Support Protocol, work in progress, Internet Engineering Task Force draft-ietf-nemo-basic-support01.txt, June 2003.

[5] R. Draves and R. Hinden. Default Router Preferences, More-Specifi c Routes, and Load Sharing, Work in Progress, Internet Engineering Task Force draft-ietf-ipv6router-selection-02.txt, June 2002.

[6] T. Ernst. Network Mobility Support Terminology, work in progress, Internet Engineering Task Force draft-ietf-nemoterminology-00.txt, May 2003.

[7] D. Jonhson, C. Perkins, and J. Arkko. Mobility support in IPv6, work in progress, Internet Engineering Task Force draft-mobileip-ipv6-24.txt, January 2003.

[8] J. Manner and M. Kojo. Mobility Related Terminology, Work in Progress, Internet Engineering Task Force draftietf-seamoby-mobility-terminology-04.txt, April 2003.

[9] N. Montavont, R. Wakikawa, T. Noel, and T. Ernst. Problem Statement of Multihomed Mobile Node, work in progress, Internet Engineering Task Force draft-montavont-mobileipmultihoming-pb-statement-00.txt, October 2003.

[10] C. Ng and J. Charbon. Multi-homing Issues in Bi-directional Tunneling, Work in Progress, Internet Engineering Task Force draft-ng-nemo-multihoming-issues-01.txt, May 2003. 